\title{
The nurses' voice of working in a newly established community based 24-hour support center for people with psychiatric disabilities
}

\author{
Ann-Christin Janlöv* , Agneta Berg \\ School of Health and Society, Kristianstad University, Kristianstad, Sweden \\ Email: *ann-christin.janlov@hkr.se
}

Received 5 March 2013; revised 6 April 2013; accepted 1 May 2013

Copyright (C) 2013 Ann-Christin Janlöv, Agneta Berg. This is an open access article distributed under the Creative Commons Attribution License, which permits unrestricted use, distribution, and reproduction in any medium, provided the original work is properly cited.

\section{ABSTRACT}

This study explores the perceptions of a small group of nurses working at a newly established 24-hour community-based service enter (SC) for users with psychiatric disability using a qualitative approach. Since the mental health reform in Sweden in 1995 where the communities (in Sweden called municipalities) were given the responsibility to establish service and support to people with severe psychiatric disabilities, they have struggled in finding suitable forms of these kinds of areas. In 2010, this led to the creation and development of a new center aiming to provide services and support based on the expressed needs of people with physchiatric disability in a community located in southern Sweden. During 2011, a total of three group interviews were performed to capture the employed nurses' perceptions of this newly established SC. The interview texts were analyzed by way of qualitative content analysis. A first reading of the interview texts revealed that the nurses' perceptions of the service center were unwaveringly positive but that their beliefs about who the specific target group were differed. The main finding was summarized by the theme: Making a difference-on an individual, professional, and organizational level. The sub themes were: 24-hour availability, unclear assignment, and preventing mental illness. The findings indicate a need for a community round-the-clock service center in this Swedish community and a more clear definition of the target group.

Keywords: Psychiatric Disability; Mental Health Nursing; Community Mental Health Service; 24-Hour Service Center; Qualitative Content Analysis

"Corresponding author.

\section{INTRODUCTION}

\subsection{Background}

The mid 1950s was the beginning of an international consensus to change the institutional mental health system to a new community based approach. Areas of focus were adequate environment, prevention, rehabilitation and social integration which are still ongoing international processes [1]. In Sweden, this change in mental health care meant deinstitutionalization through the Mental Health Reform in 1995. The objective was to support a life of inclusiveness and participation for people with psychiatric disability in the community [2]. This organizational shift meant that the communities became legally obligated to offer social support, housing, and activities for people with psychiatric disability [3].

Shortly thereafter, national reports $[4,5]$ showed that the communities had difficulty finding forms of support and service that matched the needs and desires of the users. Recent "National guidelines for psychosocial interventions for schitzophrenia or schitzophrenia-type conditions" recommend that both the health care system (county council psychiatry) and social services (communities) take action towards quality improvements in the effort to reduce the number of hospitalizations, days in hospital, homelessness, and unemployment in order to provide greater stability in the lives users with psychiatric disability [6]. The current situation has opened up for mental health staff, users, and politicians to create and implement innovative ideas for support and non-medical services.

A literature review on community mental health services/centers brought to light a sizeable amount of international and national research. Studies on mental health services centers span multiple countries and encompass an array of health services, from smaller units to large 
complexes of interconnected units offering medical treatment, various therapies, activities and even implementation of treatment programs-all named differently. This made it difficult to identify studies on non-medical support centers within community mental health services, particularly centers with around-the-clock availability. The centers in the studies seemed to be open during daytime, Monday through Friday. A systematic literature review by Catty et al. [7] highlights the problem with an imprecise nomenclature in the field. Taking these difficulties into consideration, there seems to be a lack of research focusing on community based service centers open around the clock.

\subsection{Background for the Specific Study}

The described difficulty to provide matched service to users with psychiatric disability has led to a unique initiative taken by a Swedish community. A work group consisting of community politicians, officials, and the management of the community mental health care and service was started in 2006-2007 after signs of insufficiencies within the mental health care and service where identified. Shortly thereafter, a reference group was formed with representatives from the care department, social services, private care providers, Region Skåne and users. As a result, a decision was made to establish a new nonmedical center to provide service based on users' own expressed desires for support. The support center was started year-end 2009/2010 and operated around-theclock and offered anonymity by not requiring registration or documentation. Since this was a new community service it seemed important to explore what the SC has meant to both users and nurses so far. The next step will focus on the user perception, while the aim of this present study focuses on the nurse perspective of working in a newly established community based 24-hour support center for people with psychiatric disability.

\section{METHOD}

The chosen design was an explorative, descriptive study, with a qualitative approach based on group interviews, analyzed by qualitative content analysis.

\subsection{Context}

In the larger Swedish psychiatric health care and service context of the study, care and service are provided by two systems: the county councils $(n=18)$, regulated by the Health and Medical Services Act [8] and the communities $(n=290)$, regulated by the Social Services Act [9]. The county councils provide psychiatric specialist care, emergency care, in and out-patient care. The primary care/health centers have the basic of the study for mild mental illness, while moderate and severe mental illness is referred to specialist mental health care. The communities provide housing, activities, and needs-assessed support according to the Social Service Act and legislation of support and service to users with psychiatric disability [10].

The specific context of the study was a city of an average-sized community of 50.000 inhabitants in Skåne in southern Sweden. The local community psychiatric care and service organization also encompassed two smaller communities, altogether 70.000 inhabitants. Adult psychiatric care in the city consisted of two general psychiatric care teams, psychosis team, and hospital ward for general psychiatry, for forensic psychiatric care, outpatient care and daycare. The emergency department was situated in a larger city 30 - 40 kilometers away. At the time, the community mental health service consisted of two assisted housing compounds and one meeting point with activities open during daytime hours from Monday to Friday.

The new SC was placed on the top floor of a building in the city center which already contained assisted housing. The SC was equipped with two bedrooms, "support beds", to be used a maximum of 48 continuous hours if needed. Visitors had to ring a bell to be let in. All floors were manned 24 hours a day and staff on duty were responsible for the housing floors. The housing assistance was mainly carried out during mornings and, thereafter, the nurses mainly moved their activities to the SC. Altogether, sixteen nurses were part of the staff. The head of the unit was a registered nurse responsible for the daily management. The visiting users were described as between 20 - 70 years of age. Most visitors had prior experience with mental health services, but also users without prior experience with mental health services visited. Most were men, but there were also women. An initial six-month survey indicated that 50 - 75 users contacted SC for supportive conversation. Multiple users called several times per night. Each call lasted anywhere from a couple of minutes to an hour. The support beds were utilized by approximately ten users to stay overnight.

\subsection{Participants}

A sample of employed staff $(n=16)$ at the SC was invited to take part in group interviews. Twelve staff members from day and night shifts agreed to participate, while four declined to participate due to sick leave or urgent care tasks. Eleven licensed mental practical nurses (LMPN) and one registered mental health nurse participated. Five were female and seven male. Their ages varied between 25 - 59 years (mean 41). Their previous work experience varied in length and spanned acrossmental health care, elder care, and care of adolescents with disabilities. They were all trained in Case Manage- 
ment (CM) technique, but the extent varied between 7.5 to 30 credits.

\subsection{Interviews}

Group interviews (three with four nurses) were chosen to obtain nurses' experiences through discussions about the topic. This is a strategy inspired by Kreuger and Casey [11], interviewing by planned designed group discussions. An interview guide was used and started with an overarching question, "Can you please tell me about your experiences and perceptions about the SC?” Further questions concerned their work at the SC, the target group, policy, goals, and details about user telephone calls and visits. Overall, the participants in the groups were interactive and engaged during the discussions. The second author, $\mathrm{AB}$, was a moderator in interview I, while ACJ observed and took notes. ACJ led interviews II and III while an experienced colleague observed. The nurses were interviewed between June-November 2010 in a room at the SC. The interviews lasted 120, 75 and 100 minutes. At this time, the SC had been up and running for between six to ten months. The interviews were tape recorded and transcribed by ACJ. Ethical considerations related to the study followed the Swedish law for human research [12]. Permission to conduct the study was obtained by the head of the unit. All the nurses received invitations to take part in the study as well as both written and oral information. Twelve filled out informed consent in regard to being interviewed.

\subsection{Analysis}

Qualitative content analysis was used, inspired by Graneheim and Lundman's [13] recommendations. The analysis process started with a reading of all interviews, first individually and thereafter consecutively to get a sense of the whole. Meaning units, which are lines or excerpts of discussions, were identified, condensed and abstracted into codes. Those with similar meaning content were brought together into five sub-themes of which two, after further processing, were collapsed and three sub-themes remained. Both authors reviewed the interview texts independently, discussed codes thoroughly when developing the themes and sub themes until a consensus was reached. Quotations from the three group interviews were marked I, II and III. Participants were marked in order: 1, 2, 3, and 4. A discussion excerpt between Nurse 2 and 4 can be marked (II: 2, 4) Endings of single quotations are marked //.

\subsection{Findings}

The finding is reflected in the theme: Making a difference-on an individual, professional, and organizational level, with the sub themes: Availability 24 hours a day, unclear assignment, and Preventing mental illness.

\subsection{Making a Difference on an Individual, Professional and Organizational Level}

The text revealed a strong conviction that the SC makes a difference on an individual level to users with any kind of mental illness in terms of having unique availability 24 hours a day. This appeared to make a difference in terms of the possibility of the SC being used as a meeting point, being located in the city center, having home-like venues and atmosphere, being free of charge to visit, and accessible without making an appointment. Other advantages were the user's high degree of autonomy and the option of remaining anonymous.

The staff was proud to be chosen for a position at the SC and to work in a way that makes a difference also on a professional level. Thenew position introduced a different professional way of working: free, unprejudiced, supportive and preventive. There was an evident ambition to change existing views of mental illness to one where it is considered a part of human normality. In addition, the ambition was to represent vulnerable users in contacts with powerful authorities. Ideas of how this should be done varied. On an organizational level, the SC was strongly believed to make a difference in representing something totally new, both locally and nationally. The new approach was highlighted as a unique and constantly-staffed round-the-clock support system, buffering admission to the Psychiatric emergency department (PED). However, it also emerged that the political assignment regarding SC policy and nurses' overall task was some what unclear. The intention was to welcome any person with any kind of mental illness, but when it came to practice it proved to be a challenge.

\subsection{4-Hour Availability}

The most significant with the SC was an immediate agreement in the groups about "availability around the clock" in the community. Users now had a place to go when needing someone. It provided the opportunity to get away from loneliness to security, to solve tricky situations, to make new contacts and to create a social network. Newcomers received special attention in order to fully capture their situation and need of support. This way, the nurses could prevent the users from falling between cracks. The possibility to call the SC by phone at any time was much appreciated. Many say: "now I feel calm. It feels so good that I can call you" (III: 2). There is someone that answers the phone... just that is great! (II: 2). Nights were considered a critical time with regard to anxiety and sleeping problems. A user could call several times per night when feeling anxious. Just talking to 
a nurse usually helped. The possibility to visit the SC at any time was also appreciated by the users. Always having somewhere to go, to sit down and relax, have a cup of coffee, talk to others, or just to have people around. When users felt higher degrees of anxiety the nurses were approached for a talk: "Since loneliness is a big part, they need to talk their anxiety away" (III: 3). The two support beds were considered much needed and frequently used. The beds made it possible for users to stay overnight if nurses deemed it necessary:

Users that have not slept for a couple of nights can relax and calm down and sleep (II: 2). To get help to get through a severe anxiety attack. If using the support beds, they can just open the door and see that we are there for them, so they can feel close to other people, and secure (III: 1)

Together with availability, the option to stay anonymous was viewed as the most unique aspect of the SC. This strategy aimed to attract users with fears of being stigmatized. Anonymity was possible if no formal support was requested, since no documentation was needed then:

That you can phone anonymously and don't have to expose yourself is new // We have those who want to be anonymous, not to reveal their identity. They just want to talk (III: 1, 2). There are some that absolutely don't want to come and show themselves. It is shameful. No one must know, but they want someone to talk to (II: 2).

The SC was also believed to relieve the burden for the next of kin. The discussions revealed that users' next of kin often "burn out" as a result of overwhelming them with phone calls and visits around-the-clock. Support and advice was also given when the next of kin were worried. However, the nurses emphasized that next of kin were not contacted routinely, only if the users requested it.

\subsection{Unclear Assignment}

Questions about the work directive/assignment prompted ambiguous discussions: Nobody told us that this is the goal. We had a hunch about what we wanted to do // Rather it has been: "We will see how things turn out" (I: $3,4)$. It was difficult to define what target group the SC focused on. At first, a total openness for all people was spoken of. Users with psychiatric disabilities were not predefined as a target group:

There is no target group, since you don't have to be enrolled anywhere. People who are sitting at home and need some kind of help // No more than that we shall be here for all persons that want help, but don't want to turn to psychiatric care (III: 1, 2). We don't have a definite target group. All are welcome. We have an open attitude // We have an ambition to reach people with no psychiatric contact, without being enrolled in the care system (I: 4, 5).

The discussions revealed that the desired openness was a difficult balance. For instance, they had to reject users from other communities. Moreover, users those were self-harmers or under the influence of drugs could not be handled. Other difficult situations concerned users with suicidal thoughts, or homeless people. The nurses expressed their difficulties and disagreements of the boundaries of drawing a line here:

Can you feel mentally well if you don't have any place to sleep? (III: 3) I was confronted with suicidal thoughts. We said that we cannot have this kind of problem here. But she begged that if she only could stay overnight here...she would calm down // Yes, and one man came here, smelled and had red eyes. Then you are not supposed to let the user in...but I decided to... I considered him as in need of help. You have to listen to your heart // That was a good decision. (I: 2, 3, 6)

The SC was said to relieve pressure on medical care and PED. Several users had expressed an aversion to seeking help at PED due to previously being rejected there. The nurses were convinced that the support beds had prevented several admissions. Also, community actors such as housing supporters and users' representatives had noticed a positive difference and that they could direct users to the SC. One nurse said: "Users sense that they don't have time for them at PED. There, it is all about medicine" (II: 2).

\subsection{Preventing Mental Illness}

The encounter with the users was said to be the art of their work and believed to prevent further mental illness and disability. Since vulnerability was considered one of the main problems, accepting and affirming the users as ordinary human beings was extremely important. Just being present to offer security was similarly important. The encounters, where nurses felt more like fellow peers than health care staff, were described as uncomplicated and unconventional. At the same time, it was considered very delicate work. It was challenging to show those with prior negative experiences with the traditional mental health care system that this is a new model, a new approach with new thoughts and new contacts. Some users were described as very tense upon entering the SC, but after they had sat down for a while: "one can actually see how they relax".

Is there something that is particularly difficult in your work with your group of users? You have to be incredibly responsive when you have them on the phone. It is easier when you have someone in front of you // Yes, you have to be really alert all the time // You can say something to some people to make them feel better, while you 
definitely cannot say it to others (I: 2, 4, 2).

A start in building up a relationship was to be interested in the user as a unique person, to be responsive, and to pick up on what is going on in his/her life-and to remember the core of it. Later asking questions about it was a successful way to make the user feel comfortable and cared about, and to break negative thinking:

It is important to refer back to topics in the previous conversation. "Such as, how did it go"? They have expressed surprise, "Wow, do you remember that"? They feel that we care, and the conversation gets much more personal // Yes, you can almost see how they light up. There is someone who cares, someone that has listened (III: 2, 4).

A key point in the group discussions concerned aspects of the encounter which nurses could have different opinions about such as the difficulty providing sufficient time if nurses themselves felt stressed. It could be necessary to limit phone calls at night, and a difficult balance to end. Further, it could be difficult to strike a balance between on the one hand letting a user sit down relax and just be, without demands, and on the other hand having an urge to talk, motivate or activate him/her. Thus, it was expressed as a difficult balance to accept the users' choice of not wanting to-and trying not to motivate other choices:

Some do not want to make changes. I think this is frustrating. We offer help, but despite this they don't want I // I think it can create anxiety with too many possibilities. They can't cope with it. We have to accept that. Don't give up... give hope. It is their choice (III: 4, 2).

When the discussions dealt with the difficulty of striking a balance between being supportive and creating dependence, the question of making demands came up: We do not make demands ... it does not mean that there are no strings attached. Then they would become dependent on us instead of more independent (III: 1). Some users considered the SC, "very cozy" (the others laugh), and visited too often wanting the support beds. Such patterns were a regular problem necessary to break, not to create difficulties for them to go home. Demands were viewed as necessary in their support work at the same time as it was important that visiting users did not feel pressure.

\section{DISCUSSION}

The organizational shift to give the communities the responsibility to provide care and service for mentally disabled users has been shown in Sweden to open up for some organizational innovations. As a whole, Making a difference - on an individual, professional, and organizational level was interpreted as presenting the nurses' views of the newly-established SC. They proudly viewed the SC as a national innovation. This new type of non- medical, 24-hour support evidently has advantages such as constant availability, anonymity, autonomy, family relief, prevention of relapse and hospitalization. However, disadvantages were shown in that the political assignment and target group was unclear. In addition, the lack of documentation related to anonymity could pose a risk in that nurses may lack knowledge about users' diagnoses, history and medication.

The finding, 24-hour availability, revealed the nurses' conviction that the constant opening hours was the most important resource, even more so than anonymity. What meant most to individual users was expressed as the difference of always having somewhere to phone or go when feeling the need. All nurses stressed that anxiety and loneliness reached a critical point for users, particularly at night, and contacts and visits were frequent at this hour. It is well known in mental health nursing that anxiety and insecurity often cause sleep disorders, a detrimental risk for already mentally-exposed disabled users. Burns and Firn [14], pioneers in community based psychiatric care, stress early interventions and the importance of recognizing instability in health such as anxiety, insecurity and sleep problems which can be early signs of relapse into disease. Several examples were given of users suffering from severe anxiety who had felt much better after a talk or spending a night or two at the SC.

The SC appeared to relieve the described loneliness that haunted users at night. Loneliness was shown by Nilsson et al. [15] to impact users on three levels: behaviorally, existentially, and ontologically. In their findings, users with severe mental disease living alone at home felt: shame over their illness; loneliness as a pain accompanied by anxiety; and defenselessness and loss, which was visible as self-isolation. Their own residence did not feel like home and they had a longing to belong. It was interpreted as "loneliness of want and longing". The fact that loneliness is detrimental to both physical and mental health is known through research that supports links between feelings of loneliness/social isolation and increased risk of morbidity and mortality [16]. Having relief close at hand in a place like the SC reduces users' suffering and distress and reduces pressure on PED, which should motivate other communities enough to start offering similar support.

The availability of the SC was also improved by being non-medical, free of charge, based on user anonymity, and not subject to traditional patient-nurse treatment terms. This high availability was an intended strategy to attract people that currently are without psychiatric care or service contacts with various degrees of mental disability in order to prevent any mental conditions from getting worse. People who had been identified by social workers as having severe mental illness were, in Rosenberg [17], found to avoid psychiatrically-defined services due 
to the fear of being stigmatized as mentally ill or disabled and, consequently, labeled as deviant and not normal. This label comes with the added cost of humiliation, loss of status and discrimination [18]. User avoidance of mental health care services is understandable and user anonymity can be legitimized.

As indicated in the finding, unclear assignment, it was difficult to define the target group. This unclearness could also be found in the finding preventing mental illness in the way nurses carried out their daily work. These findings begged the question as to how the target group was defined and handled within the team. All people that were identified as suffering from some kind of mental distress were said to be welcome. However, this is a view that differed some what from the official assignment which expressly stated to accept only people who were considered to be suffering from some form of psychiatric disability? The difficulty with clearly defining the target group was further observed with users with ongoing drug abuse, suicidal thoughts, and self-harm behaviors. In a newly published national report [19], the difficulty in defining psychiatric disability was made evident. The lack of a clear and consistent definition on a national level carries a risk that county councils, communities, the work group/team as well as the single nurse make up their own definitions. However, the result showed that welcoming all had proved to be impossible and there was some disagreement within the team about which individuals to reject. Such unclearness can create conflicts within the work team and feelings of inadequacy on the individual level leading to qualms of conscience [20] and, at worst, burnout [21]. Beyond the quandary of whom to accept or reject, another consequence of the difficulty to define the target group was how to strike a balance between demands and permissiveness for the users.

The conviction of having a new way of treating users in comparison to the traditional mental health care approach was shown in preventing mental illness. The encounter was strongly believed to relieve and heal mental distress and prevent progression of illness and disease. Some aspects stood out. It was seen as a particular challenge to approach users with prior negative experiences from mental health contacts in the past and to convince them to trust that this is a "new model, a new approach with new thoughts and new contacts". Users as patients' in the psychiatric health care system have often experienced violation and powerlessness when being approached. Lilja and Hellzén [22] found that when patients recounted past experiences of psychiatric care, much concerned a struggle to maintain dignity in discriminating and ostracizing environments. The nurses told about users' experiences of rejection and subsequent reluctance to seek psychiatric care. Rejection as a neglected phe- nomenon in psychiatric nursing has been found as ways to understand the professional role and responsibility in terms of inadequate staffing [23]. According to ideas of Lögstrup [24], trusting someone means to expose your self and thereby risk being rejected. Abused trust causes mistrust-negative encounters and experiences, which the nurses at SC felt were a challenge to change. That positive encounters can have a "healing" effect was shown in a Swedish study [25]. A sample of long-term sick-listed patients $(n=5802)$ were asked about positive and negative encounters in the health care system, and the effects of these encounters on their ability to return to work. Those who had experienced positive encounters and felt respected $(n=3327)$ experienced a significantly improved ability to return to work in comparison to those who did not feel respected $(n=79)$. Those who in addition felt wronged $(n=993)$ claimed to be more impeded from returning to work compared to those who did not feel wronged $(n=410)$. Consequently, it seems as merely the way of approaching users with mental problems can promote their mental health and prevent illness and disease. Such healing encounters 24-hours a day can make a difference - even rendering admission to medical care altogether unnecessary.

Steps were taken throughout the research process to ensure trustworthiness of the findings [26]. Attempting to maximize researcher credibility, as recommended by Patton [27], qualified people with professional competence and awareness of the sensitivity of the subject matter conducted the study. Similarly, testing the interview guide was a similar endeavor. The participants spoke openly in the group interviews which generated rich data for the research focus. By the third interview, similar topics were repeated and saturation was considered reached. That group interviews and verbatim transcriptions were conducted by the authors $\mathrm{ACJ}$ and $\mathrm{AB}$ should strengthen the findings. Excerpts from the interviews were also presented to validate that the themes represented the participants' experiences/views of the SC. Limitations and threats to credibility is that not all staff members participated. The risk with the group interviews is that staff members may have held back in their statements since they were dependent on each other. Through descriptions of the participants, context, methods of data collection and analysis process, readers should be able to judge the transferability of the findings.

\section{CONCLUSION}

The findings of this study indicate that a 24-hour community based service centre has the potential to make a positive difference on the three levels found in the study: the individual user level since loneliness and anxiety often peak at night, the nurses' professional level trough 
the new unconventional way of working, and the organisational level by the SC being designed to support user needs around-the-clock. The findings also indicate that when establishing new services, the target group needs to be more distinctively identified.

\section{REFERENCES}

[1] Novella, E.J. (2010) Mental health care in the aftermath of deinstitutionalization: A retrospective and prospective view. Health Care Analysis, 18, 222-238. doi:10.1007/s10728-009-0138-8

[2] Markström, U. (2003) The Swedish mental health reform. Ph.D. Thesis, Umeå University, Umeå.

[3] Markström, U., Lindqvist, R. and Sandlund, M. (2009) Case management for people with psychiatric disabilites in rural Sweden; experiences from implementation of a national policy. European Journal of Social Work, 12, 495-508. doi:10.1080/13691450902930787

[4] National Board of Health and Welfare and the County Administrative Board (2005) The contributions of municipalities for persons with mental illnesses. Final Report from a National Supervision. Socialstyrelsen Och Länsstyrelserna, Stockholm.

[5] SOU (2006) Ambitions and responsibilities. National scheme for development of social contributions to persons with mental illnesses and mental disabilities. Socialstyrelsen, Stockholm.

[6] National Board of Health and Welfare (2011) National Guidelines for Psychosocial Interventions for Schitzofrenia or Schitzofrenia-Type Conditions-Support for Governance and Management. Socialstyrelsen, Stockholm.

[7] Catty, J.S., Burns, T., Comas, A. and Poole, Z. (2008) Day centres for severe mental illness (review). The cochrane collaboration. The Cochrane Library, 3, 1-16. doi:10.1002/14651858.CD001710

[8] SFS (1982) Health and medical services act. Hälso-Och Sjukvårdlagen, Stockholm.

[9] SFS (2001) Social services act. Socialdepartementet, Stockholm.

[10] SFS (1993) Act of support and service for the disabled. Socialdepartementet, Stockholm.

[11] Krueger, R.A. and Casey, M.A. (2009) Focus groups. A practical guide for applied research. 4th Edition, Sage Publications, Thousand Oaks.

[12] Codex (2012) Codex-Rules and guidelines. http://www.codex.vr.nu

[13] Graneheim, U.H. and Lundman, B. (2004) Qualitative con- tent analysis in nursing research: Concepts, procedures and measures to achieve trustworthiness. Nurse Education Today, 24, 105-112. doi:10.1016/j.nedt.2003.10.001

[14] Burns, T. and Firn, M. (2005) Socially-based psychiatric care: A guide for practitioners. Studentlitteratur, Lund.

[15] Nilsson, B., Nåden, D. and Lindström, U.Å. (2008) The tune of want in the loneliness melody-Loneliness experienced by people with serious mental suffering. Scandinavian Journal of Caring Sciences, 22, 161-169. doi:10.1111/j.1471-6712.2007.00508.x

[16] Cacioppo, J.T., Hawley, L.C., Crawford, L.E., et al. (2002) Loneliness and health: Potential mechanisms. Psychosomatic Medicine, 64, 407-417.

[17] Rosenberg, D. (2009) Psychiatric disability in the community. Surveying the social landscape in the post-deinstitutional era. Ph.D. Thesis, Umeå University, Umeå.

[18] Link, B.G. and Phelan, J.C. (2001) Conseptualising stigma. Annual Review of Sociology, 27, 363-385. doi:10.1146/annurev.soc.27.1.363

[19] National Board of Health and Welfare (2012) Taking stock of needs. An inventory of a group of persons with mental disabilities. Socialstyrelsen, Stockholm.

[20] Glasberg, A.L., Eriksson, S. and Norberg, A. (2008) Factors associated with "stress of conscience" in healthcare. Scandinavian Journal of Caring Sciences, 22, 249-258. doi:10.1111/j.1471-6712.2007.00522.x

[21] Glasberg, A.L., Eriksson, S. and Norberg, A. (2006) Burnout and "stress of conscience" among healthcare personnel. Journal of Advanced Nursing, 57, 392-403. doi:10.1111/j.1365-2648.2007.04111.x

[22] Lilja, L. and Hellzén, O. (2008) Former patients' experience of psychiatric care: A Qualitative investigation. International Journal of Mental Health Nursing, 17, 279286. doi:10.1111/j.1447-0349.2008.00544.x

[23] Hem, M.H. and Heggen K. (2004) Rejection-A neglected phenomenon in psychiatric nursing. Journal of Psychiatric and Mental Health Nursing, 11, 55-63. doi:10.1111/j.1365-2850.2004.00687.x

[24] Lögstrup, K.E. (1956/1997) The ethical demand. Notre Dame, University of Notre Dame Press, London.

[25] Lynöe, N., Wessel, M., Olsson, D., Alexandersson, K. and Helgesson, G. (2011) Respectful encounters and return to work: Empirical study of long-term sick-listed patients' experiences of Swedish health care. BMJ Open, 1, Article ID: e000246. doi:10.1136/bmjopen-2011-000246

[26] Lincoln, Y.S. and Guba, E.G. (1985) Naturalistic inquiry. Sage Publications, London.

[27] Patton, M.Q. (2002) Qualitative research \& evaluation methods. 3rd Edition, Sage Publications, Thousand Oaks. 